\title{
Experimentell- ökologische Untersuchungen über die limitierenden Faktoren des mikrobiellen Ölabbaues im marinen Milieu
}

\author{
WILFRIED GUNKEL \\ Biologische Anstalt Helgoland, Meeresstation, Helgoland
}

\begin{abstract}
Experimental-ecological investigations regarding the limiting factors of microbial oil degradation in the marine environment. This contribution deals with model experiments of bacterial oil degradation. It considers oils which are likely to pollute the marine environment. The amounts of different oils resisting bacterial degradation were determined quantitatively. Numbers of oil decomposing bacteria were counted using a MPN (mostprobable-number) technique with mineral oils as the only carbon source. The influence of the following factors was determined: inorganic nitrogen- and phosphate salts, enrichment cultures, easily decomposable organic substances (other than oil), and temperature. In most experiments freshly sampled seawater with its natural content of marine bacteria was used. The special distribution of oil decomposing bacteria in oil-water mixtures was investigated. The possible influence of these factors on the degradation of mineral oils in the sea is discussed.
\end{abstract}

\section{EINLEITUNG}

Es gibt viele Beziehungen zwischen Mikroorganismen - insbesondere Bakterien und Mineralölen. Mehrere tausend Arbeiten sind auf diesem Gebiet publiziert worden. Zusammenfassende Darstellungen mit Literatursammlungen beispielsweise bei BEERStecher (1954), Zobell (1946, 1950, 1962), Sharpley (1961), Fuhs (1961), Foster (1962), Treccani (1965), Málex \& Schwartz (1966). So gilt als sicher, daß die Mineralöle in flachen Randmeeren entstanden sind, wobei den Bakterien eine beträchtliche Rolle in der Transformation des Ausgangsmaterials zukam (z. B. EMERY 1960). Praktisch alle organischen Substanzen können, sofern die Bedingungen dazu günstig sind, durch Bakterien abgebaut werden, so auch Mineralöle und Olprodukte. Meist sind diese Aktivitäten unerwünscht und können zu großen finanziellen Einbußen führen. Einige Beispiele: Wuchs von Mikrootganismen in den Treibstoffen von Düsenflugzeugen verbunden mit Verstopfen der Filter (Brennstoffzufuhr) und Tankkorrosion. Beides führt zu unsicheren Betriebsbedingungen. Desgleichen können angegriffen werden beispielsweise Kühlöle, Asphaltstraßen, medizinische Salben und Emulsionen sowie Isolationsmaterialien. Olabbauende Bakterien sind eine häufige Ursache der Innenkorrosion und damit für das Undichtwerden von Lagertanks verantwortlich. Große Aktivitäten ölabbauender Bakterien sind dagegen erwünscht einmal bei der mikrobiellen Proteinsynthese aus Olen, zum anderen bei der Beseitigung von Olverunreinigungen. 
Die Verwendung von Mineralölen und seinen Produkten ist in den letzten Jahren gewaltig angestiegen. Damit haben sich auch die Olverunreinigungen, hervorgerufen durch Unfälle als auch durch normale Handhabung, stark vermehrt. Die Skala der Verunreinigungen reicht von Tankschiffunfällen, bei denen mehrere tausend Tonnen $O 1$ in das Gewässer gelangen können, wie beispielsweise im Jahre 1965 durch die $\mathrm{Ha}-$ varie der „Anne-Mildred-Brövig“ in der Nordsee, zu den Unfällen von Olversorgungsfahrzeugen und Pipelinebrüchen bis zum Verschütten geringer Mengen beim Umfüllen. Undichte Heizöllagertanks können größere Mengen in den Boden treten lassen. Bei der Reinigung von Tanks und Motoren sowie mit Industrieabwasser und aus Reparaturwerkstätten können beträchtliche Mengen Ol in die Gewässer und mit den Flüssen in das Meer gelangen. Die schädigenden Einflüsse können groß sein und ganze Lebensgemeinschaften vernichten, wobei die durch Verölung verendeten Vögel zwar am augenfälligsten sind, aber nicht die am stärksten betroffene Gruppe zu sein braucht. Häufig jedoch werden trotz stärkerer zeitweiser Verölung im Endeffekt nur geringfügige Veränderungen der Tier- und Pflanzenwelt festgestellt, die aber, obwohl keine Abtötung stattfindet - zum Beispiel durch starke geschmackliche Beeinflussung des Fischfleisches und damit Ungenießbarkeit für die menschliche Ernährung -, zu großen finanziellen Verlusten führen können.

Ober die tatsächliche schädigende Wirkung der Verölung, über die Verweildauer des Oles im Boden sowie über die Rückhaltekraft des Bodens sind im Gegensatz zu ihrer Bedeutung bisher nur wenige Untersuchungen durchgeführt worden. Trotz der großen Anzahl Veröffentlichungen über Beziehungen zwischen Mikroorganismen und Ol ist praktisch nichts bekannt über die Geschwindigkeit des Olabbaues unter natürlichen Verhältnissen und über die Frage der Giftigkeit der Olabbauprodukte. Es bestehen stark unterschiedliche Ansichten über diese Probleme, und die Diskussion darüber ist im höchsten Grade emotionell belastet (GAsionowski 1965). Aus diesem Grunde erschien es notwendig, Untersuchungen über die Faktoren anzustellen, die einen OOlabbau begrenzen.

Der Ursprung meines Interesses an den Fragen des Olabbaues lag darin, daß mit dem Ol eine neue Kohlenstoffquelle in das Meerwasser gelangt, die jetzt eine Rolle im Stoffkreislauf des Meeres spielt. Durch die Aktivität von Meeresbakterien werden Ole abgebaut. Es ist zu erwarten, daß sie sich in irgendeiner Weise darauf einstellen, anpassen und daß Populationsverschiebungen auftreten können. Darüber hinaus stellt das Meerwasser ein ausgezeichnetes System dar, um ganz allgemein Modellvorstellungen zum Olabbau zu gewinnen. Anorganische Nährsalze und leicht abbaubare organische Substanzen sind nur in äußerst geringen Mengen vorhanden, und die Bakterienzahl ist gering. Dadurch ist es leicht möglich, durch Variation der Bedingungen, wie beispielsweise der anorganischen Nährsalze, deren Einfluß zu untersuchen.

\section{METHODIK}

Die umfangreichen Untersuchungen, die zur Methodik durchgeführt wurden, müssen einer gesonderten Veröffentlichung vorbehalten bleiben. Hier seien die angewandten Methoden lediglich kurz erwähnt. 
Die Abbauversuche wurden in 1-1-Glasstopfenflaschen angesetzt. Die eingewogene Olmenge betrug bei den meisten Untersuchungen $50 \mathrm{mg} /$ Flasche, die Flüssigkeitsmenge $500 \mathrm{ml} /$ Flasche. Falls Stickstoff- und Phosphorsalze zugefügt wurden, war die Endkonzentration die gleiche wie in dem weiter unten aufgeführten Nährmedium für die Bestimmung der ölabbauenden Bakterien. Zur Bestimmung der jeweiligen Bakterienzahlen wurden zu verschiedenen Zeiten aus einer Flasche geringe Probenmengen entnommen. Für die Bestimmung des Ölabbaues dagegen wurde eine größere Anzahl Flaschen angesetzt und nach den bestimmten Versuchszeiten je 1 beziehungsweise 2 Flaschen einer Reihe mit $10 \mathrm{ml}$ konzentrierter Salzsäure versetzt und damit der Abbau durch Abtötung der Bakterien unterbrochen.

(a) Bestimmung des Ölgehaltes: Die jeweiligen Restmengen an Ơl wurden nach Extraktion mit Tetrachlorkohlenstoff mit Hilfe von Infrarotspektrophotometrie im Laboratorium der BP-Olgesellschaft, Hamburg, nach der Methode von Simrad et al. (1951) quantitativ bestimmt.

(b) Bestimmung der Bakterienzahlen: Die Anzahlen zum Olabbau befähigter Bakterien wurden mit einer Verdünnungsmethode, der sogenannten MPN (most-probable-number) Methode (Am. Public Health Assoc., 1955), bestimmt. Der verwandte Nährboden hatte folgende Zusammensetzung:

$\begin{array}{lc}\text { gealtertes Seewasser } & 750 \mathrm{ml} \\ \text { destilliertes Wasser } & 250 \mathrm{ml} \\ \mathrm{NH}_{4} \mathrm{Cl} & 0,5 \mathrm{~g} \\ \mathrm{~K}_{2} \mathrm{HPO}_{4} & 0,5 \mathrm{~g} \\ \mathrm{Na}_{2} \mathrm{HPO}_{4} & 1,0 \mathrm{~g} \\ \mathrm{pH} & 7,6\end{array}$

Die Sterilisation von Seewasser einerseits und den Stickstoff- und Phosphorsalzen im destillierten Wasser andererseits wurde getrennt vorgenommen. Der $\mathrm{pH}$-Wert wurde mit Natronlauge eingestellt. Als alleinige Kohlenstoffquelle wurde pro Einzelfläschchen mit $10 \mathrm{ml}$ Nährlösung je 1 Tropfen des entsprechenden sterilen OOles zugefügt. Die Bebrütung erfolgte bei $18^{\circ} \mathrm{C}$ drei Wochen lang. Für die „Keimzahlbestimmung" wurde wie in früheren Untersuchungen (z. B. GuNKel 1964) die Gußplattenmethode mit dem Nährboden 2216 E herangezogen.

Es interessierten Mineralöle, die tatsächlich Verunreinigungen verursachen, wie zum Beispiel die Heizöle EL, M, S; Petroleum, Schwergasöl, Irak Rohöl und Slop cut. Mineralöle sind äußerst komplizierte Stoffgemische. Aus naheliegenden Gründen wurden daher die chemischen Umwandlungsstufen und die Endprodukte des Abbaues nicht untersucht, sondern rein quantitativ die Restmenge Öl. Reinkulturen von Bakterien wurden nicht verwandt, sondern frisch geschöpttes Seewasser mit seiner natürlichen Bakterienpopulation.

\section{ERGEBNISSE}

Ausgangspunkt der Untersuchungen waren die in Abbildung 1 dargestellten Verhältnisse. Die abgebildeten 5 Flaschen waren 4 Wochen lang bei $18^{\circ} \mathrm{C}$ aufbewahrt worden. Sie enthielten von links nach rechts: Seewasser ohne Zusätze; Seewasser mit 


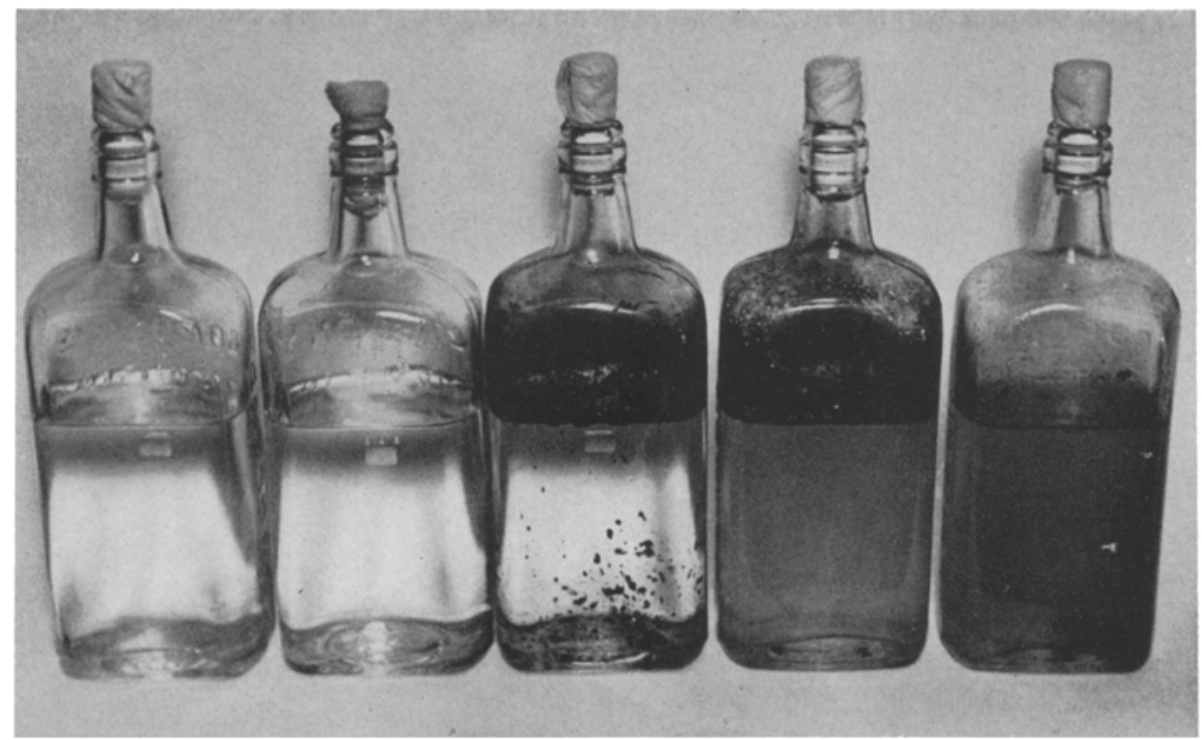

Abb. 1: Bakterienwuchs in Seewasser beziehungsweise in Seewasser mit Irak-Rohöl mit weiteren Zusätzen nach einer Standzeit von 4 Wochen bei $18^{\circ} \mathrm{C}$. Die starke Trübung in der 4. und 5. Flasche von links zeigt Bakterienwuchs auf Kosten des Oles an. Weitere Erklärungen im Text

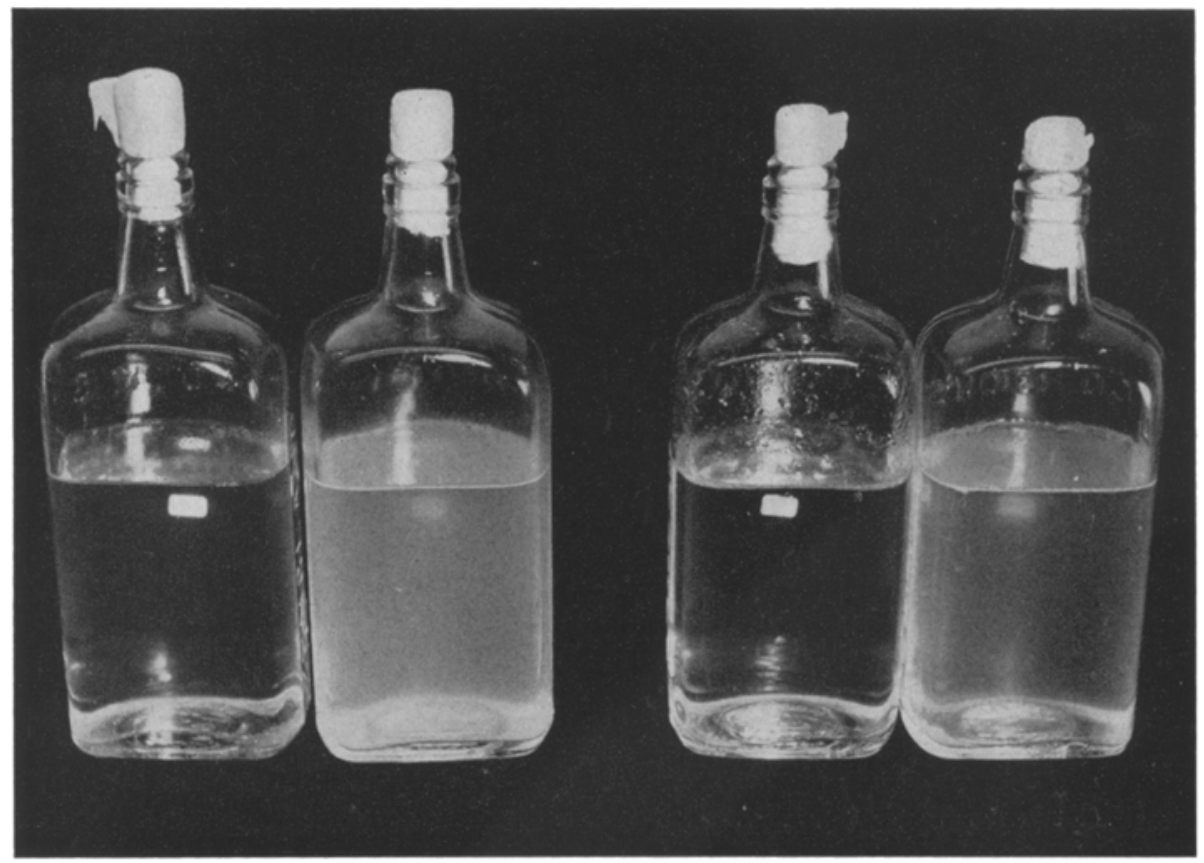

Abb. 2: Fördernde Wirkung von N- und P-Salzen auf den Olabbau. Das linke Flaschenpaar enthält Seewasser und Petroleum, das rechte Seewasser und Vakuumgasöl. Der rechten Flasche jeden Paares waren jeweils N- und P-Salze zugesetzt worden. Die Standzeit betrug 4 Wochen bei $18^{\circ} \mathrm{C}$. Die Trübung zeigt Bakterienwuchs an 
N- und P-Salzen; Seewasser und 500 mg Irak-Rohöl; Seewasser, 500 mg Irak-Rohöl sowie N- und P-Salze; Seewasser, $500 \mathrm{mg}$ Irak-Rohöl, N- und P-Salze sowie $1 \mathrm{~g}$ eines am Strand von List/Sylt aufgelesenen alten sandigen Olfladens.

In den ersten beiden Flaschen war rein optisch keine Veränderung festzustellen. Auch das Wasser der dritten Flasche blieb klar, bei genauerer Betrachtung konnten jedoch einzelne weiße Bakterienflocken an der Unterseite der das Wasser bedeckenden

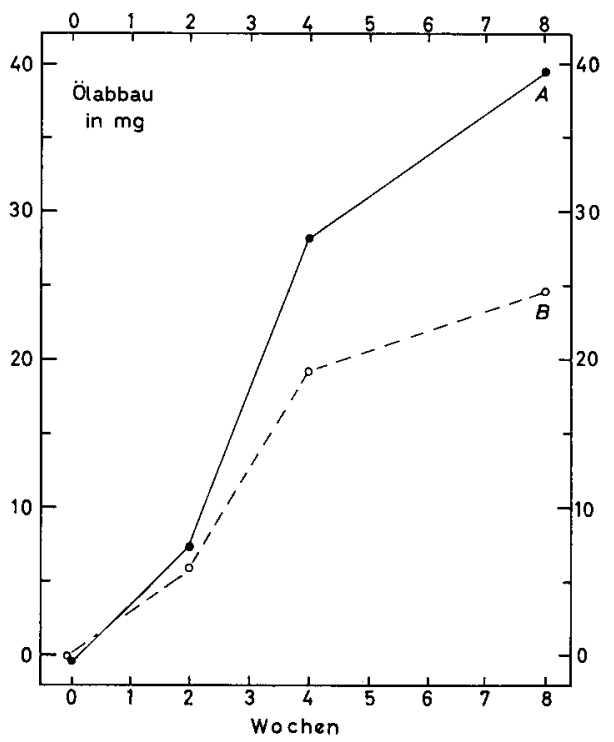

Abb. 3: Abbau von Schwergasöl durch eine natürliche Bakterienpopulation. Schwergasöleinwaage $50 \mathrm{mg}$. A: Seewasser und Schwergasö1; B: Seewasser, Schwergasöl und N- und P-Salze

OOlschicht festgestellt werden. Die vierte Flasche dagegen zeigte eine sehr starke Trübung des Inhaltes; ein heftiger Bakterienwuchs auf Kosten des Oles hatte stattgefunden. Noch stärker war die fünfte Flasche getrübt; das OOl an den oberen Wandungen war fast völlig verschwunden.

Abbildung 2 zeigt den fördernden Einfluß der N- und P-Salze beim Abbau von Vakuumgasöl (linkes Flaschenpaar) und Petroleum (rechtes Flaschenpaar) im Seewasser. Die rechte Flasche eines jeden Paares ist mit N- und P-Salzen versetzt, die linke ohne diese Zusätze. Nur in den Flaschen mit N- und P-Salzen ist eine starke Vermehrung der Bakterien festzustellen.

In Abbildung 3 wird der quantitative Verlauf des Abbaues von Schwergasöl ersichtlich. Die Einwaage betrug jeweils $50 \mathrm{mg}$ Ol. Die Kurve B wurde bei Seewasser ohne weitere Zusätze erhalten, die Kurve A zeigt den Abbau in Flaschen, die zusätzlich N- und P-Salze enthielten.

Die entsprechenden Bakterienzahlen sind in Abbildung 4 als Anzahl ölabbauender Bakterien pro $\mathrm{ml}$ als Logarithmen eingetragen. Dem höheren Abbau des Ansatzes mit N- und P-Salzen entspricht eine beträchtliche höhere Anzahl Bakterien. 


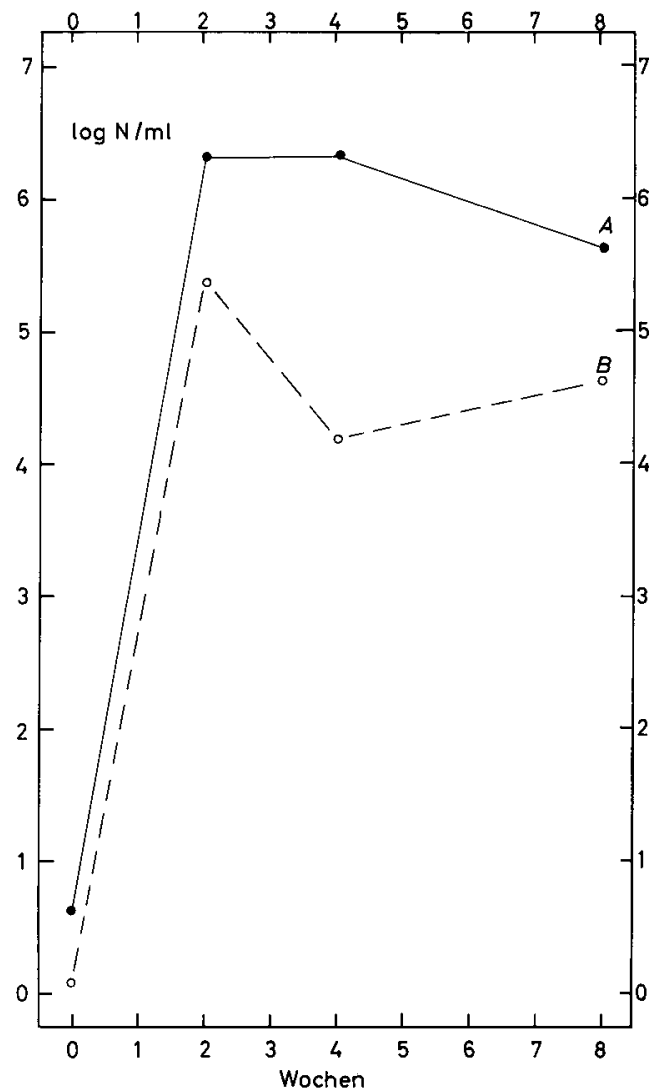

Abb. 4: Zunahme ölabbauender Bakterien während des Abbaues von Schwergasöl entsprechend der Abbildung 3. A: Seewasser, Schwergasöl und N- und P-Salze; B: Seewasser und Schwergasöl

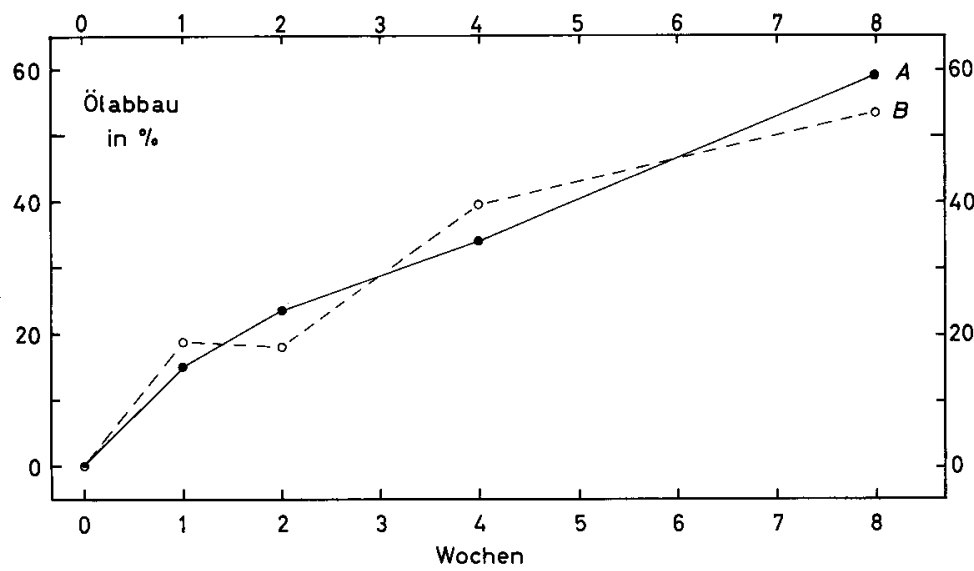

Abb. 5: Abbau von Vakuumgasöl durch eine natürliche Bakterienpopulation eines SeewasserSandsediment-Gemisches. A: Sediment und Vakuumgasöl; B: Sediment, Vakuumgasöl und Nund P-Salze. Versuch 34/(1965) 
Die Abbildung 5 zeigt den Abbau von Vakuumgasöl in einer Versuchsreihe, bei der pro Flasche $700 \mathrm{~g}$ Sand von der Niedrigwassergrenze und $250 \mathrm{ml}$ Seewasser eingefüllt wurden. Im Gegensatz zu sämtlichen Ansätzen mit Seewasser wurde hier praktisch kein Unterschied zwischen den Proben mit und ohne N- und P-Salzen gefunden.

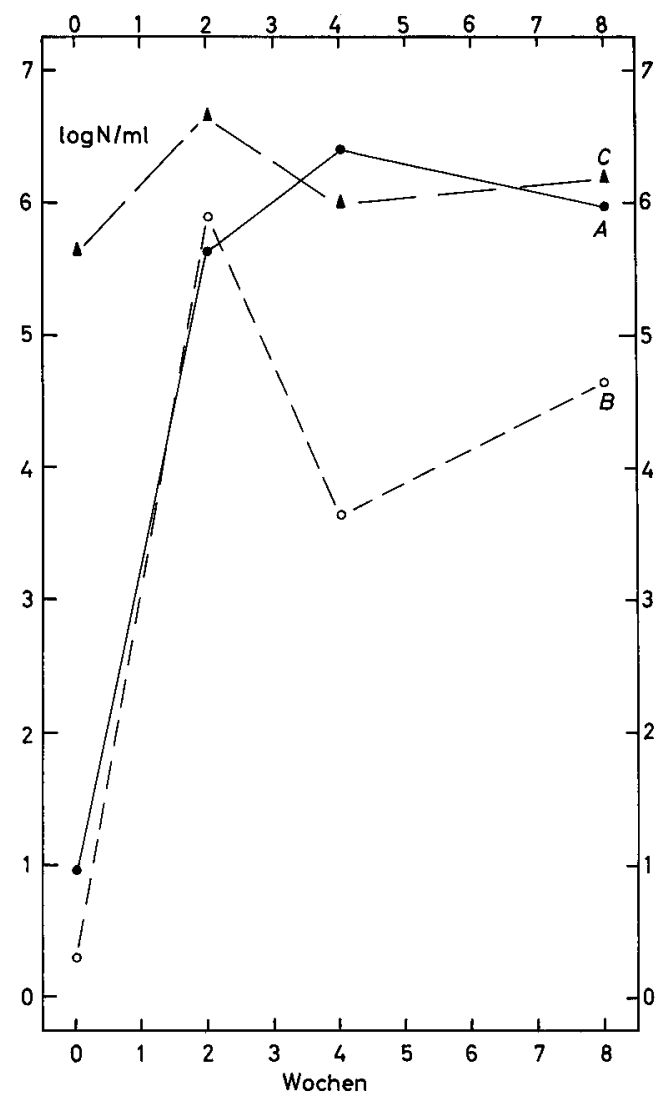

Abb. 6: Zunahme ölabbauender Bakterien während des Abbaues von Vakuumgasöl. A: Seewasser, Vakuumgasöl und N- und P-Salze; B: Seewasser und Vakuumgasöl; C: gleicher Ansatz wie A, jedoch wurde eine Anreicherungskultur zugefügt

Während bei sämtlichen bisher angeführten Untersuchungen lediglich die im frisch geschöpften Seewasser vorhandenen Bakterien für einen Abbau zur Verfügung standen, wurden in einigen Versuchen auch Flaschen mit einer Anreicherungskultur ölabbauender Bakterien versetzt. Hierbei wurden jedoch im Endeffekt keine höheren Abbauwerte festgestellt. In Abbildung 6 sind die Anzahlen Bakterien der Ansätze: Seewasser und $\mathrm{Ol}(\mathrm{B})$, Seewasser, N- und P-Salze und OOl (A) sowie Seewasser, N- und P-Salze, Ol und Anreicherungskultur (C) dargestellt. Es ist deutlich zu erkennen, daß durch Bakterien der Anreicherungskultur zwar bei Versuchsbeginn eine höhere Anzahl öl- 
abbauender Bakterien anwesend war, jedoch später kein Unterschied mehr zu den Zahlen des Ansatzes Seewasser, Oll und N- und P-Salze bestand.

Abbildung 7 zeigt den Einfluß von gleichzeitig vorhandenen, leicht abbaubaren organischen Substanzen auf den Olabbau. Untersucht wurde der Abbau von jeweils $50 \mathrm{mg}$ Heizöl $M$ in Seewasser. Während eine Reihe (A) keine Zusätze erhielt und zu einer weiteren Reihe N- und P-Salze in der üblichen Konzentration gegeben wurde (C), wurden bei B 2,5 g Pepton und 0,5 g Hefeextrakt und bei $\mathrm{D}$ neben 2,5 g Pepton und $0,5 \mathrm{~g} \mathrm{Hefeextrakt} \mathrm{wiederum} \mathrm{N}$ - und P-Salze zugefügt. Es ist deutlich erkennbar, daß in den beiden letztgenannten Fällen durch die gleichzeitig vorhandenen leicht abbaubaren organischen Substanzen der Beginn des Olabbaues verzögert wird, denn noch

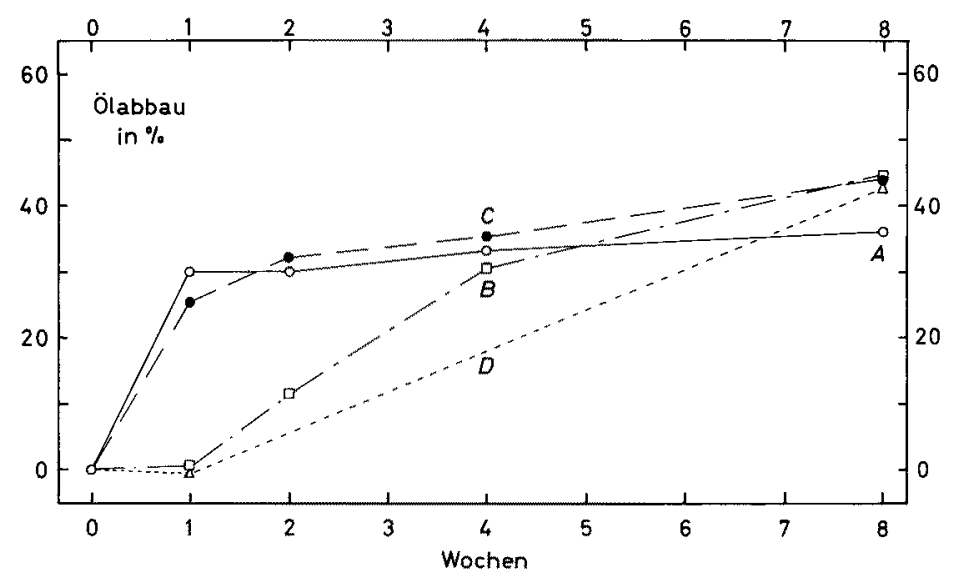

Abb. 7 : Einfluß von leicht abbaubaren organischen Substanzen auf den Abbau von Heizöl $M$ im Seewasser. A: Heizöl M; B: Heizöl M, 2,5 g Pepton und 0,5 g Hefeextrakt; C: Heizöl M und $\mathrm{N}$ - und P-Salze; D: Heizöl $\mathrm{M}, \mathrm{N}$ - und P-Salze, $2,5 \mathrm{~g}$ Pepton und $0,5 \mathrm{~g}$ Hefeextrakt. Versuch Nr. 22/(1965)

nach einer Woche ist die Ausgangskonzentration vorhanden. Eine entsprechende Entwicklung wurde bei der gleichzeitig durchgeführten Bestimmung der bakteriologischen Gruppen gefunden. Bei den Reihen B und D überwiegen zunächst die Saprophyten, bei den Reihen $A$ und $C$ die Olabbauer.

In Abbildung 8 ist der Einfluß der Temperatur auf den Abbau von Heizöl $M$ bei $2^{\circ} \mathrm{C}$ und $18^{\circ} \mathrm{C}$, welches normalerweise die niedrigste und höchste Temperatur in der Nordsee ist, dargestellt. Zusätzlich wurde eine Versuchsreihe bei $30^{\circ} \mathrm{C}$ angesetzt. Untersucht wurde wiederum der Abbau in frisch entnommenem Seewasser mit seiner natïrlichen Bakterienpopulation. Alle Flaschen wurden mit $\mathrm{N}$ - und P-Salzen angereichert. Der starke Einfluß der Temperatur ist deutlich und klar sichtbar. Während bei $2^{\circ} \mathrm{C}$ nach 2 Wochen Einwirkungszeit erst $2,5 \%$ der Einwaage abgebaut worden sind, sind es bei $18^{\circ} \mathrm{C}$ bereits $17 \%$ und bei $30^{\circ} \mathrm{C}$ sogar $27 \%$, das heißt die 10 fache Menge des $2^{\circ} \mathrm{C}$-Wertes. Dieses Verhältnis verschiebt sich stark mit der Zeit. Nach 8 Wochen Bebrütung wird bei $2^{\circ} \mathrm{C}$ die Einwaage auf mehr als die Hälfte der Menge, 
die bei $30^{\circ} \mathrm{C}$ abgebaut wurde, reduziert. Die abgebauten Mengen bei $2^{\circ} \mathrm{C}$ und $18^{\circ} \mathrm{C}$ verhalten sich nach 8 Wochen sogar wie $3: 4$.

Als weiterer untersuchter Faktor sollen einige Versuche mit einer Umwälzvorrichtung wenigstens kurz erwähnt werden. Je $50 \mathrm{mg}$ der Heizöle El, M und S und $500 \mathrm{ml}$ Seewasser, versetzt mit N- und P-Salzen, wurden in 1-I-Glasstopfenflaschen eingewogen, in eine Umwälzvorrichtung eingespannt und kontinuierlich bis $\mathrm{zu} 8$ Wochen langsam bewegt, so daß im Laufe einer Umdrehung alles $\mathrm{Ol}$ mit dem Wasser in innige

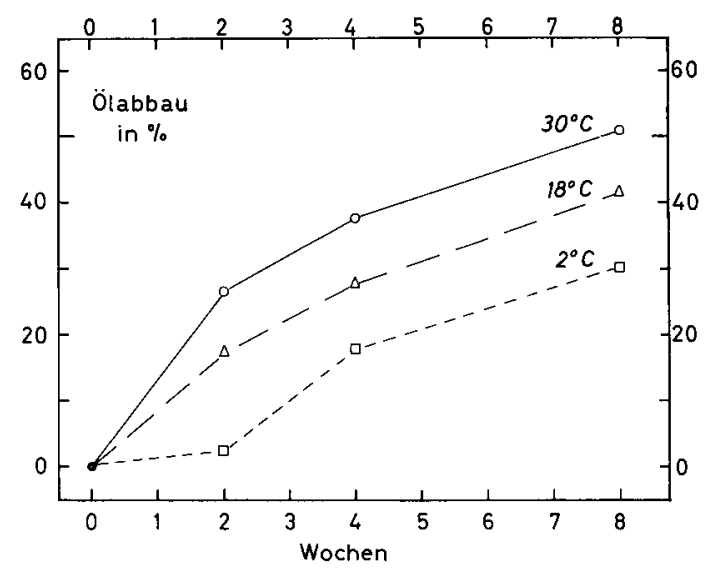

Abb. 8: Einfluß verschiedener Temperaturen auf den Abbau von Heizöl $M$ in Seewasser. Versuch Nr. 35/(1965)

Berührung kam. Eine gleiche Reihe wurde ohne Umwälzung aufbewahrt. In allen Fällen war ein stärkerer Abbau bei den umgewälzten Flaschen festzustellen. Außerdem wurde auch hier deutlich, daß, je höher der Siedepunkt des Oles, desto geringer der Abbau war. Nachstehend die erhaltenen 2-Wochen-Werte:

$\begin{array}{ll}\text { Heizöl S } & =\text { kein Abbau nachweisbar } \\ \text { Heizöl S und Umwälzung } & =7 \% \text { Abbau } \\ \text { Heizöl M } & =17 \% \text { Abbau } \\ \text { Heizöl M und Umwällzung } & =34 \% \text { Abbau } \\ \text { Heizöl EL } & =33 \% \text { Abbau } \\ \text { Heizöl EL und Umwälzung } & =60 \% \text { Abbau }\end{array}$

Die räumliche Anordnung von ölabbauenden Bakterien in Ol-Wassergemischen ist in den Abbildungen 9 bis 11 dargestellt. Es handelt sich um Ansätze mit $\mathrm{N}$ - und P-Salzen, die 16 Tage lang bei $18^{\circ} \mathrm{C}$ bebrütet worden waren. Die Abbildungen 9 und 10 zeigen die Besiedlung von Heizöl-M-Tröpfchen. Das große zentrale Gebilde ist Heizöl; bei Abbildung 9 ist optisch auf den Rand des Tröpfchens, bei Abbildung 10 auf die Oberfläche eingestellt worden. Deutlich erkennbar ist die bevorzugte Besiedlung der Grenzfläche Ol-Wasser. Ein ganz anderes Bild tritt auf bei den Ansätzen, die als Kohlenstoffquelle Petroleum enthielten (Abb. 11). Es haben sich große Bakterienmassen gebildet. Der Rest des vorhandenen Petroleums ist zu feinen Kügelchen emulgiert, die als helle überstrahlte Gebilde erkennbar sind. 


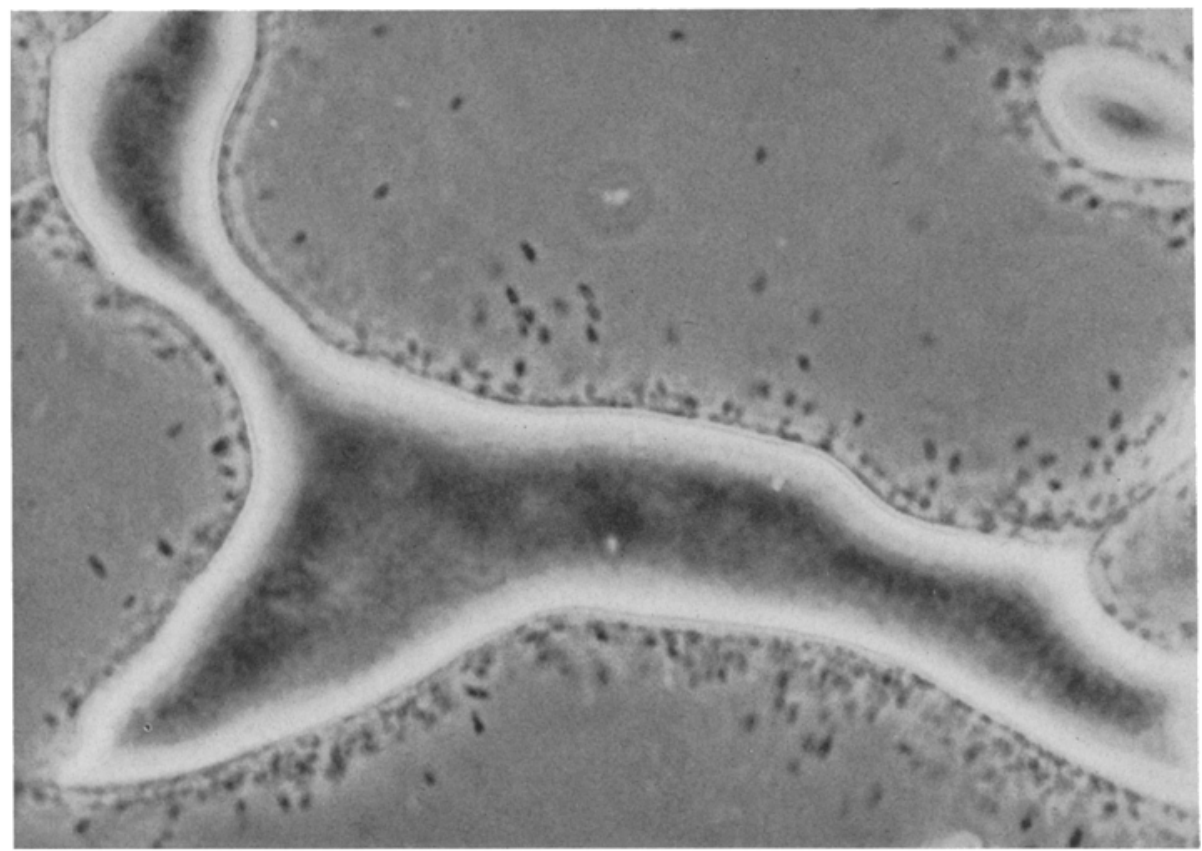

Abb. 9: Mikroskopische Aufnahme von ölabbauenden Bakterien in einem Gemisch von Heizöl $M$ und Wasser; Scharfeinstellung auf den Rand des Öltropfens

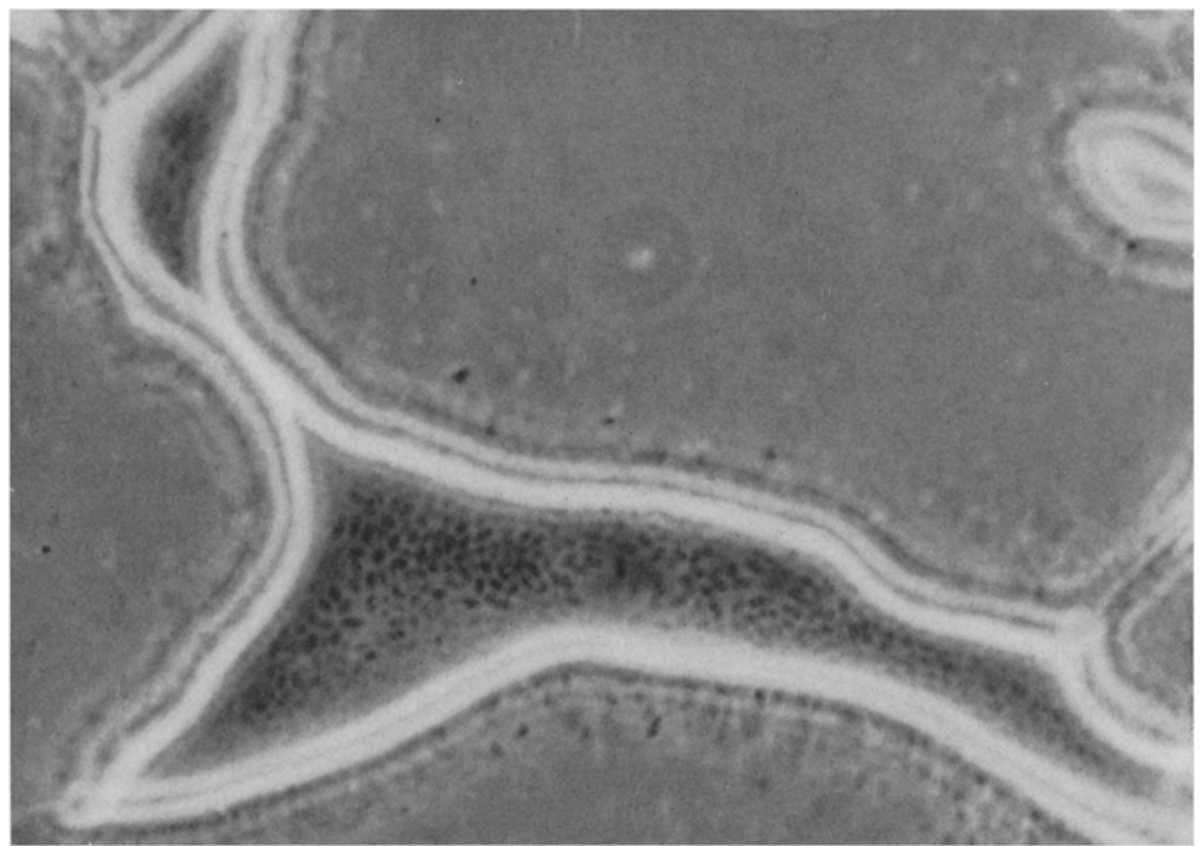

Abb. 10: Gleiches Objekt wie Abbildung 9, jedoch Scharfeinstellung auf die Oberfläche des Oltropfens 


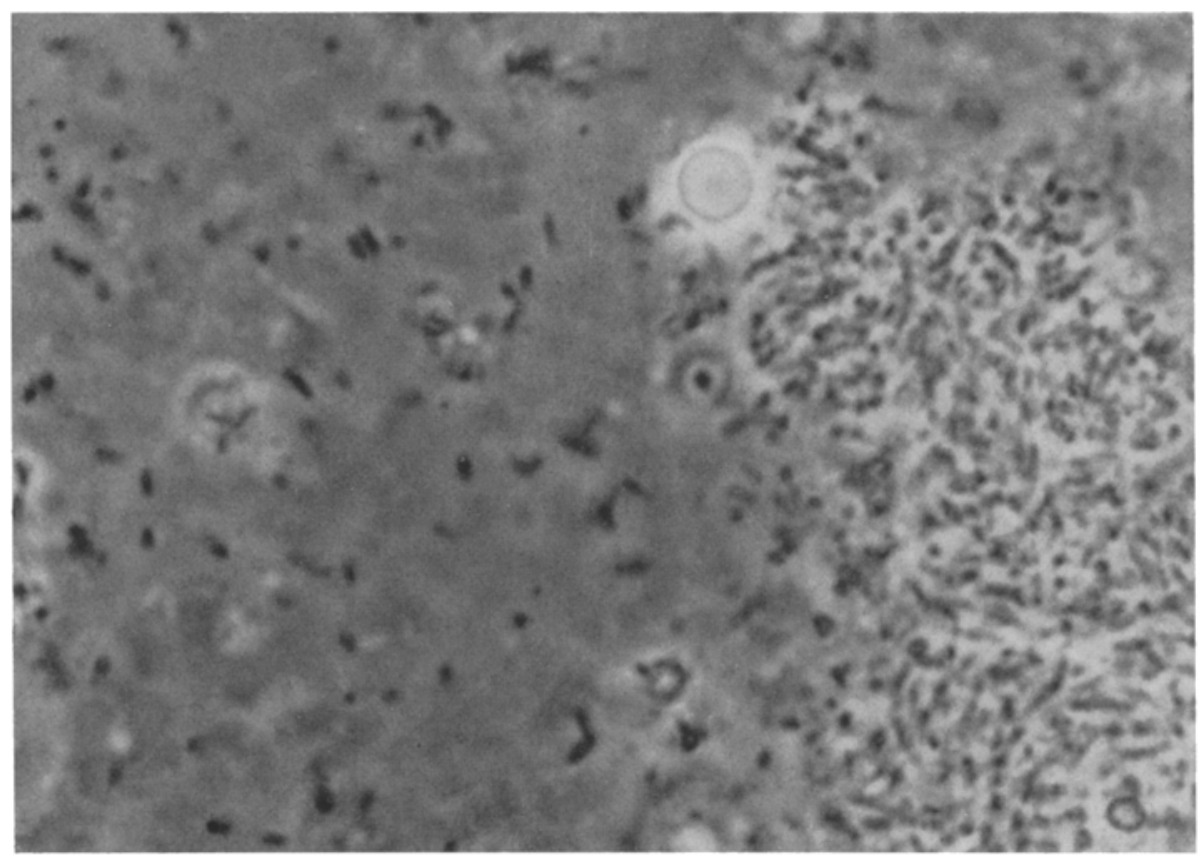

Abb. 11: Mikroskopische Aufnahme von ölabbauenden Bakterien aus einem Abbauversuch mit Petroleum

\section{DISKUSSION}

Die Untersuchungen im Seewasser haben in allen Fällen ergeben, daß die Zugabe von anorganischen Stickstoff- und Phosphorsalzen den Olabbau steigert; dies gilt sowohl für die Geschwindigkeit des Abbaues als auch für den absoluten Wert. Gleichzeitig steigt die Anzahl der ölabbauenden Bakterien um ein Vielfaches gegenüber den nicht mit diesen Salzen angereicherten Ansätzen. Selbstverständlich sind diese Salze im Seewasser vorhanden, ihre Konzentration ist jedoch sehr gering. Folgende Tabelle zeigt die in zwei Versuchen bestimmten Werte:

\begin{tabular}{lcc}
\hline Nährsalze & Versuch $12 / 1963$ & Versuch 5/1964 \\
& $\mu \mathrm{g} / \mathrm{l}$ & $\mu \mathrm{g} / \mathrm{l}$ \\
\hline $\mathrm{NO}_{2}$-Stickstoff & 13,5 & 17,4 \\
$\mathrm{NO}_{3}$-Stickstoff & 104 & 261 \\
$\mathrm{NH}_{4}$ und Aminogruppen-Stickstoff & 116 & 174 \\
$\mathrm{PO}_{4}$-Phosphat & 19,1 & 40,8
\end{tabular}

Es waren also insgesamt etwa $230 \mu \mathrm{g} / 1$ (bzw. $450 \mu \mathrm{g} / \mathrm{l}$ ) gebundener Stickstoff und $20 \mu \mathrm{g} / \mathrm{l}$ (bzw. $40 \mu \mathrm{g} / \mathrm{l}$ ) Phosphor vorhanden. 
$\mathrm{Da}$ aus allen Versuchen hervorgeht, daß der Menge der zur Verfügung stehenden $\mathrm{N}$ - und P-Salze für den Ölabbau eine große Bedeutung zukommt, sollen dazu einige theoretische Überlegungen angestellt werden: Die Nährsalze werden für den Aufbau der Leibessubstanz der Bakterien benötigt. Wir finden Stickstoff vor allen Dingen in den Aminogruppen der Aminosäuren und Phosphate in den Nukleinsäuren. Laut Angaben in der Literatur haben Bakterien einen Wassergehalt von etwa $80 \%$. Vom Trockengewicht sind etwa $50 \%$ Kohlenstoff, etwa $10 \%$ Stickstoff und etwa 3,2\% Phosphor. Wenn wir von der Annahme ausgehen, daß ein Bakterium ein spezifisches Gewicht von 1 hat (in Wirklichkeit ist es geringfügig schwerer) und im aktiven Stadium ein Volumen von $1 \mu^{3}$ einnimmt, enthalten $10^{9}$ Bakterien $1 \mathrm{mg}$ Feuchtsubstanz; dem entspricht $200 \mu \mathrm{g}$ Trockensubstanz und darin $100 \mu \mathrm{g}$ Kohlenstoff, $20 \mu \mathrm{g}$ Stickstoff, 6,4 $\mu \mathrm{g}$ Phosphor.

Die Versuche haben gezeigt, daß die durchschnittliche Maximalzahl der ölabbauenden Bakterien in Abbauversuchen bei $10^{6} / \mathrm{ml}$, das heißt 10\%/1 lag. Berücksichtigen wir weiterhin, daß fast stets die Anzahlen der Saprophyten, die wahrscheinlich die ersten Abbauprodukte der Olabbauer weiterverwerten, etwa eine Zehnerpotenz höher lagen, daß die anorganischen Salze nur bis zu einer bestimmten unteren Grenzkonzentration im Medium aufgenommen werden konnten, und daß häufig nur eine sich vergrößernde Population deutlich aktiv ist, so wird klar, warum die Stickstoff- und Phosphorsalze einen derart begrenzenden Faktor darstellen. Interessant ist in diesem Zusammenhang, daß bei einer Analyse von Elbwasser, entnommen bei Hamburg, Konzentrationen an Stickstoff- und Phosphorsalzen auftraten, die im Vergleich zu Versuch 5 (1964) 17-beziehungsweise $13 \mathrm{mal}$ größer waren.

Keinen Einfluß zeigt die Zugabe von N- und P-Salzen bei einer Versuchsreihe mit sandigem Litoralsediment (Abb. 5). In Sedimenten sind stets beträchtlich größere Mengen an $\mathrm{N}$ - und P-Salzen vorhanden als im Wasser. Limitierend dürfte hier die $\mathrm{O}_{2}$-Versorgung sein, die unter diesen Versuchsbedingungen nur schwer experimentell steuerbar ist. In den Versuchen führte Sauerstoffmangel zu begrenzten anaeroben Zonen mit Schwefelwasserstoffbildung und zur Ausfällung von Hydrotroilit. Die Genauigkeit dieser Versuche litt etwas darunter, daß organische Substanzen des Sandes als Öl miterfaßt wurden (ca. $3 \mathrm{mg} / 100 \mathrm{~g}$ Sand). Außerdem war es mit Schwierigkeiten verknüpft, das $\mathrm{Ol}$ in den verschiedenen Ansätzen in gleicher Weise zu verteilen.

Über den Einfluß von Anreicherungskulturen - ein Beispiel ist in Abbildung 6 dargestellt - können keine allgemeingültigen Aussagen gemacht werden. Im Endeffekt erfolgt hier kein stärkerer Abbau als ohne Anreicherungskultur; in einem Fall (Abb. 1), in dem ein Stück eines alten Olfladens vom Strand, sozusagen mit einer natürlichen Anreicherungskultur, verwandt wurde, kam es jedoch deutlich zu einem stärkeren Abbau. Hier spielen möglicherweise eine ganze Fülle von Möglichkeiten eine Rolle, die ihre Ursachen in den Bedingungen der Ansätze, den Bakterienpopulationen, in ihrer art- und leistungsmäßigen Zusammensetzung und der chemischen Zusammensetzung der verschiedenen Öle haben können. Alle Ursachen kann ich hier nicht diskutieren; es sei aber insbesondere hingewiesen auf das in der Mikrobiologie häufig zu findende Prinzip der Aquifinalität, das besagt, daß eine Populationsendzahl nicht von der Menge der ursprünglich vorhandenen Organismen, sondern einzig und allein von den äußeren Faktoren, wie beispielsweise Nahrung und begrenzende Faktoren, bestimmt 
wird. Die Ursache liegt in den ungeheuren Vermehrungspotenzen der Bakterien. Diese Potenzen kommen nur bei den seltensten Gelegenheiten und auch dann nur kurzfristig zur Entfaltung, da sie zwangsläufig bald limitiert werden.

Der Zufügung von Anreicherungskulturen könnte in drei Fällen eine Bedeutung als Stimulans zukommen:

(1) Wenn im Milieu keine ölabbauenden Bakterien vorhanden sind.

(2) In der ersten Phase des Abbaues (in den ersten Tagen nach der Verölung) würde die zu einer merkbaren Ölreduzierung führende Bakterienzahl schneller erreicht werden. Hierzu wären jedoch Bakterienpopulationen erforderlich, die optimal an die betreffenden Bedingungen angepaßt sind. Hätten die natürlicherweise vorliegenden Bakterien auch nur einen geringen Wuchsvorteil, der sich in einer kürzeren Generationszeit äußern würde, dann wäre die Anreicherungskultur nach wenigen Generationen im Hintertreffen.

Die Zugabe von im Labor unter unnatürlichen Bedingungen angezüchteten Reinkulturen erscheint auch auf Grund dieser Uberlegung als Hilfsmittel für die Steigerung eines Abbaues bei einer Verölung im natürlichen Lebensraum sinnlos. Versuche in dieser Richtung bei Kläranlagen, Ingolstadt, hatten auch tatsächlich keinen Erfolg.

(3) In Abbildung 1 war in der mit einer "natürlichen Anreicherungskultur" (dem alten Olrest vom Strand) versetzten Flasche eine deutliche Steigerung gegenüber anderen Ansätzen festzustellen. Bei diesem, den Ansätzen zugefügten Ơl handelte es sich um Irak Rohöl, also um die gesamte Skala der Kohlenwasserstoffe. Höhersiedende Olanteile, insbesondere Asphalte werden erfahrungsgemäß besonders schwer abgebaut. Es kann bei diesem Ansatz nicht entschieden werden, ob die im natïrlichen Seewasser vorhandenen ölabbauenden Bakterien in der Lage waren, diese Substanzen abzubauen. Die Trübung in der vierten Flasche könnte lediglich vom Abbau der niedriger siedenden Anteile herrühren. Mit dem Ollrest vom Strand, der keine niedrigsiedenden Anteile mehr enthielt, waren mit Sicherheit Spezialisten für höhersiedende Anteile zugefügt worden.

Diesen Fragen kommt erhebliche praktische Bedeutung zu. So ist offen, ob nicht die Zufügung von höher konzentrierten Emulgatoren (die einen hohen Anteil an niedrigsiedenden Kohlenwasserstoffen aufweisen und gegen Verölungen eingesetzt werden) unter Umständen die für den natürlichen Abbau wesentlichen Bakterien, welche die Fähigkeit besitzen, höhersiedende Anteile abzubauen, abtöten. Vorversuche in dieser Richtung zeigten zumindest bei einigen Emulgatoren eine hohe Giftigkeit gegenüber Bakterien.

Neben dem OOl vorhandene, leicht abbaubare organische Stoffe, wie zum Beispiel Pepton und Hefeextrakt, führen in allen Fällen zumindest in der ersten Zeit zu einer starken Reduktion des OOlabbaues beziehungsweise es vergeht längere Zeit, in der überhaupt kein Ólabbau stattfindet (Abb. 7). Während ohne diese Substanzen im Versuch Nr. 22/1965 bereits nach einer Woche etwa $30 \%$ der Einwaage abgebaut waren, war bei Vorhandensein dieser Stoffe noch kein Olabbau feststellbar. Die Saprophyten nehmen bedeutend stärker zu als die Ölabbauer. Diesem Ergebnis kommt eine größere praktische Bedeutung zu. Es muß erwartet werden, daß zum Beispiel bei künstlichem Abwasser, das gleichzeitig Oll enthält, kein Olabbau stattfindet, bevor nicht die übrigen organischen Substanzen abgebaut wurden. Sollte dann noch Sauerstoffmangel hinzu- 
kommen und sich das Ol nicht an der Oberfläche, sondern an Partikeln im Wasser befinden, so würde es - auch nach Entfernung der anderen organischen Substanzen kaum zu einem Ólabbau kommen.

Auch niedere Temperaturen haben einen stark verzögernden Einfluß auf den O1abbau. Es muß daher im Winter mit einer bedeutenden Verzögerung des Olabbaues gerechnet werden. Bei häufigen Verölungen könnte es im Winter daher trotz optimaler Abbaubedingungen zu einer Anreicherung des Oles kommen.

Die mikroskopischen Aufnahmen zeigen, daß die Bakterienbesiedlung von OlWassergemischen an der Grenzfläche Wasser-Ol vor sich geht. Dies dürfte eine der Ursachen dafür sein, daß hochviskose Öle sehr langsam abgebaut werden. Petroleum bildet emulgierende Stoffe; es wird daher durch die Bakterien fein verteilt und leicht abgebaut. Die Bakterien konnten lediglich in der wäßrigen Phase festgestellt werden. Kürzlich (HEYER 1966) wurde berichtet, daß ein Wachstum der Bakterien auch in der Olphase möglich ist. Auch hier beschränkt sich das Wachstum auf die Grenzfläche beziehungsweise auf die der Grenzfläche benachbarten Zonen.

Die Untersuchungen wurden nicht mit Reinkulturen von Bakterien und nicht mit chemisch definierten Substanzen durchgeführt, da beabsichtigt war, Modelluntersuchungen durchzuführen, die weitgehend den natürlichen Gegebenheiten entsprachen. Bei einer Beurteilung der Ergebnisse müssen jedoch folgende Tatsachen kritisch im Auge behalten werden: (1) Die quantitative Olbestimmung unterscheidet nicht zwischen verschiedenen Fraktionen beziehungsweise der Unzahl chemischer Verbindungen, die zum Beispiel ein Heizöl M ausmachen. Eine solche Unterscheidung ist unmöglich. Möglich und wahrscheinlich ist, daß der abgebaute Teil chemisch nicht mit dem Restöl identisch ist. (2) Die Ergebnisse der Experimente können selbstverständlich nicht ohne weiteres auf die Verhältnisse in der freien Natur übertragen werden. Zum Beispiel sind die Oberflächen (Glaswände) und die Wasserbewegungen mit ihren Vermischungsvorgängen in beiden Fällen verschieden.

Trotzdem sind die Ergebnisse innerhalb der Versuchsreihen vergleichbar und in ihren Relationen in gewissem Sinne übertragbar. Um die natürlichen Bedingungen besser zu simulieren, ist mit Großversuchen in jeweils $1 \mathrm{~m}^{2}$ großen künstlichen Tümpeln mit 1001 Wasserinhalt begonnen worden.

\section{ZUSAMMENFASSUNG}

1. Die Vermehrungsrate ölabbauender Bakterien und die Intensität des Ölabbaus wird im Seewasser durch Zugabe anorganischer Stickstoff- und Phosphorsalze wesentlich gefördert. In unzureichenden Mengen stellen diese Salze einen sehr wirksamen limitierenden Faktor dar.

2. In den daraufhin untersuchten Sedimenten findet keine Förderung des Olabbaus durch Zufügung anorganischer Stickstoff- und Phosphorsalze statt, obwohl beträchtlich höhere Mengen dieser Salze als im Seewasser vorhanden waren. Vermutlich findet hier eine Begrenzung des Abbaues durch Sauerstoffmangel statt; jedenfalls deutet die Bildung von schwefelwasserstoffhaltigen Zonen auf eine derartige Möglichkeit hin. 
3. Die Zugabe von Anreicherungskulturen führte nicht generell zu einem verstärkten Olabbau.

4. Gleichzeitig vorhandene, leicht abbaubare organische Substanzen hemmen den OIabbau sehr stark.

5. Niedrige Wassertemperaturen haben einen stark verzögernden Einfluß auf den Olabbau.

6. In Heizöl-Wasser-Gemischen besiedeln die Bakterien fast ausschließlich die Grenzflächen O1-Wasser.

7. Bakterien sind in der Lage, in Petroleum-Wasser-Gemischen das Petroleum weitgehend zu emulgieren und dadurch die Abbauvorgänge zu beschleunigen.

Die Untersuchungen wurden durch die BP-Benzin- und Petroleumgesellschaft fnanziell unterstützt. Die quantitativen Oibestimmungen wurden im Raffinerie-Laboratorium der BP in Hamburg durchgeführt. Den Herren Dr. Voss, Dr. Francke und Dr. Winguth bin ich zu großem Dank verpflichtet. Herrn Dr. Grlebricht und Herrn Harms von der Biologischen Anstalt Helgoland danke ich für die Bestimmung des Phosphor- beziehungsweise des gebundenen Stickstoffgehaltes in den Wasserproben. Für wertvolle, gewissenhafte Mitarbeit sage ich insbesondere meinem Technischen Assistenten, Herrn TreKEL, der auch die Abbildungen anfertigte, meinen herzlichen Dank.

\section{ZITIERTE LITERATUR 1}

Beerstecher, E., 1954. Petroleum microbiology. Elsevier, Amsterdam, 376 pp.

Foster, J. W., 1962. Hydrocarbons as substrates for microorganisms. Antonie van Leeuwenboek 28, 241-274.

Furs, J. W., 1962. Der mikrobielle Abbau von Kohlenwasserstoffen. Arch. Mikrobiol. 39, 374-422.

Gasionowski, K., 1965. Wissenschaft statt Vermutung. Oel 9, 1-5.

Gunkel, W., 1963. Daten zur Bakterienverteilung in der Nordsee. Veröff. Inst. Meeresforsch. Bremerh. (Sonderbd 1), 80-89.

- 1964. Einwirkungen des kalten Winters 1962/63 auf die Bakterienpopulationen vor Helgoland. Helgoländer wiss. Meeresunters. 10, 246-256.

Heyer, J., 1966. Beobachtungen über das Wachstum von Bakterien in nichtwässrigen Medien. In: Vorträge des internationalen Symposiums „Erdölmikrobiologie“, Brno (CSSR), 5.-7. 10. 1964. Hrsg. von I. Málek \& W. Schwartz. Akad.-Verl., Berlin, 227-229.

Málek, I. \& SCHWARTz, W. (Hrsg.), 1966. Vorträge des internationalen Symposiums „Erdölmikrobiologie", Brno (CSSR), 5.-7. 10. 1964. Akad.-Verl., Berlin, 240 pp.

Pearson, E. A. (Ed.), 1964. Advances in water pollution research. Proceedings of the international conference held in London, 1962. Pergamon pr. Oxford, Vol. 3, 1-437.

Pollutrons marines par les microorganismes et les produits petroliers. Symposium de Monaco, Avril 1964. Ed.: Commission Internationale pour l'Exploration Scientifique de la Mer Méditerranée, Monaco. Sécrétariat Général de la Commission, Paris 1965, 384 pp.

Sharpley, J. M., 1961. Applied petroleum microbiology. Memphis, Tenn., Buckman Labs, Inc., 196 pp.

Simrad, R. G., Hasegawa, I., Bandaruk, W. \& Headington, C. E., 1951. Infrared spectrophotometric determination of oil and phenols in water. Analyt. Cbem. 23, 1384-1387.

STANDARD methods for the examination of water, sewage, and industrial wastes, 1955. 10th ed. Am. Public Health Ass., New York, 522 pp.

Treccani, V., 1965. Microbial degradation of aliphatic and aromatic hydrocarbons. $Z$. allg. Microbiol. 5, 332-341.

1 Dieses Verzeichnis erhebt keinen Anspruch auf Vollständigkeit. Aufgenommen wurden insbesondere zusammenfassende Darstellungen, durch deren umfangreiche Literaturverzeichnisse der gesamte Problemkreis Mikroben-Erdöl erfaßt sein dürfe. 
ZoBell, C. E., 1946. Action of microorganisms on hydrocarbons. Bact. Rev. 10, 1-49.

- 1950. Assimilation of hydrocarbons by microorganisms. Adv. Enzymol. 10, 443-486.

- 1964. The occurrence, effects and fate of oil pollution in the sea. In: Advances in water pollution research. Vol. 3. Ed, by E. A. Pearson. Pergamon pr., Oxford, 85.

\section{Diskussion im Anschluß an den Vortrag GunkeL}

RHEinheimer: Könnte die hemmende Wirkung von verschiedenen organischen Stoffen auf den Mangel an organischen Nährstoffen beziehungsweise Wirkstoffen zurückzuführen sein?

GunkeL: Nicht in unseren Versuchen, da der Olabbau bei Zufügung leicht abbaubarer organischer Verbindungen zwar erst nach längerer Zeit in Gang kam, jedoch nach 8 Wochen den gleichen Wert erreichte.

RHEINHEIMER: Können außer Kohlenwasserstoffen noch andere organische Stoffe als Nahrung verwendet werden?

Gunkel: Diese Frage dürfte wohl nur für Reinkulturen eindeutig zu beantworten sein. Bei Populationen erhalten wir starke Verschiebungen je nach Angebot der Nahrungsstoffe. Das heißst, wenn leicht abbaubare Substanzen vorhanden sind, vermehren sich die Saprophyten unter Abbau dieser Substanzen, während die Zahl der Olabbauer nicht zunimmt.

HUEck: Haben Sie Gelegenheit gehabt zu überprüfen, ob auch anaerober Abbau stattfindet?

GunKel: Wir mußten uns zwangsläufig auf den aeroben Abbau beschränken. Anaerober $\mathrm{Ab}$ bau ist wiederholt in der Literatur beschrieben worden, zum Beispiel von Ivanov (1962) und Simakova (1962). Die Abbaugeschwindigkeit ist bedeutend geringer als unter vergleichbaren aeroben Bedingungen. Anaerober Ólabbau wurde auch von Zonell \& Prokop (Z. allg. Mikrobiol. 6, 143, 1966) beschrieben. (Anmerkung während der Drucklegung.) Ob Reinkulturen von sulfatreduzierenden Bakterien Ol abbauen können, ist nach der Literatur (z. B. PosT'GATE 1965, Bact. Rev. 29, 425) fraglich.

MEYERS: Did you observe any specific bacterium involved?

Gunkel: Einige Reinkulturen wurden isoliert, jedoch wurden keine taxonomischen Untersuchungen angestellt.

MEYers: Have you seen any incidence of yeast or filamentous fungi in your culture filtrates? Gunkel: Wir haben eine größere Anzahl von Proben mikroskopisch untersucht. Wir entwickelten dazu mit Herrn Dr. THon, Göttingen, eine neue Methode, die eine einwandfreie Antwort gibt. In keinem Fall konnten wir in den Abbauversuchen Hefen oder Pilze feststellen, stets nur Bakterien; Kulturversuche zur Anreicherung und Isolation von Hefen oder Pilzen wurden nicht durchgeführt.

VAuk: Wie wirkt sich das OOl im Seewasser auf die nicht am Abbau beteiligten Bakterien aus? Sind hier Vergiftungserscheinungen aufgetreten?

GUNKEL: Wir untersuchten stets die natürlich vorkommenden Bakteriengemische und erfaßten rein zahlenmäßig Bakterien, die zum Olabbau befähigt sind bzw. in anderen Ansätzen die Saprophyten (Keimzahl). Hierbei konnten keine Vergiftungserscheinungen festgestellt werden. Ganz im Gegensatz dazu waren die zur Olbekämpfung verwandten Emulgatoren $a b$ einer bestimmten Konzentration deutlich toxisch, wenn auch in den meisten Fällen nach einiger Zeit eine Anpassung der Population erfolgte. Die Giftigkeit des Oles auf Reinkulturen wurde von uns nicht untersucht.

SAVAGE: Have you any information on the effect of agitation on the decomposition of oils?

Gunkel: Ja. Wir führen mehrere Reihen von Untersuchungen durch, in denen die Flaschen der Abbauversuche in eine Umwälzmaschine eingespannt waren. In allen Fällen war der Abbau deutlich gesteigert. Häufig war die doppelte Menge abgebaut. 